\title{
Is Lutikizumab, an Anti-Interleukin-1 $\alpha / \beta$ Dual Variable Domain Immunoglobulin, efficacious for Osteoarthritis? Results from a bayesian network meta-analysis
}

\author{
Ziqin Cao $\mathbb{D}^{1},{ }^{1}$ Yajia Li $\left(\mathbb{D},{ }^{2}\right.$ Wanchun Wang, ${ }^{1}$ Shuo Jie, ${ }^{1}$ Xuantao Hu, ${ }^{1}$ Jian Zhou, ${ }^{1}$ Tong Wu, \\ Dilihumaer Aili, ${ }^{1}$ Zeling Long, ${ }^{3}$ Yihan $\mathrm{Li}^{4}{ }^{4}$ Pengcheng Dou $\mathbb{D}^{1},{ }^{1}$ and Ren Wu ${ }^{1}{ }^{1}$ \\ ${ }^{1}$ Department of Orthopedics, The Second Xiangya Hospital, Central South University, Changsha, Hunan 410011, China \\ ${ }^{2}$ Department of Dermatology, Xiangya Hospital, Central South University, Changsha, China \\ ${ }^{3}$ Department of Orthopedic Surgery, Mayo Clinic Rochester, USA \\ ${ }^{4}$ Department of Orthopedic Surgery, University of California, Davis Medical Center, USA
}

Correspondence should be addressed to Pengcheng Dou; doupengcheng@csu.edu.cn and Ren Wu; wurenraul@hotmail.com

Received 31 May 2020; Revised 20 September 2020; Accepted 9 October 2020; Published 5 November 2020

Academic Editor: Fabiano Bini

Copyright (c) 2020 Ziqin Cao et al. This is an open access article distributed under the Creative Commons Attribution License, which permits unrestricted use, distribution, and reproduction in any medium, provided the original work is properly cited.

\begin{abstract}
Objective. Most guidelines recommend the use of nonsteroidal anti-inflammatory drugs (NSAIDs), duloxetine, and tramadol for the nonoperative treatment of osteoarthritis (OA), but the use of them is limited by the tolerability and safety concerns. Lutikizumab is a novel anti-IL- $1 \alpha / \beta$ dual variable domain immunoglobulin that can simultaneously bind and inhibit IL-1 $\alpha$ and IL- $1 \beta$ to relieve the pain and dysfunction symptoms. We conducted this network meta-analysis to comprehensively compare the clinical efficacy and safety of lutikizumab with other drugs recommended by guidelines. Methods. We conducted a Bayesian network and conventional meta-analyses to compare the efficacy and safety of lutikizumab with other traditional drugs. All eligible randomized clinical trials, in PubMed, CNKI, EMBASE, and Web of Science databases, from January 2000 to January 2020, were included. The Cochrane risk of the bias assessment tool was used for quality assessment. Pain relief, function improvement, and risk of adverse effects (AEs) were compared in this study. Results. 24 articles with 11858 patients were included. Duloxetine (DUL) had the largest effect for pain relief (4.76, 95\% CI [2.35 to 7.17]), and selective cox-2 inhibitors (SCI) were the most efficacious treatment for physical function improvement (SMD 3.94, 95\% CI [2.48 to 5.40]). Lutikizumab showed no benefit compared with placebo for both pain relief (SMD 1.11, 95\% CI [-2.29 to 4.52]) and function improvement (SMD 0.992, 95\% CI [-0.433 to 4.25]). Lutikizumab and all other drugs are of favorable tolerance for patients in the treatment of OA compared with placebo. Conclusions. Lutikizumab, the new anti-Interleukin- $1 \alpha / \beta$ dual variable domain immunoglobulin, showed no improvement in pain or function when compared with placebo. Selective cox-2 inhibitors and duloxetine remain the most effective and safest treatment for OA. More high-quality trials are still needed to reconfirm the findings of this study.
\end{abstract}

\section{Introduction}

Osteoarthritis (OA) is the most common form of joint disease, usually affecting load-bearing joints such as hip and knee joints [1]. Approximately 302 million people suffer from OA worldwide every year [2]. OA can lead to local pain and joint stiffness in its early stages and can cause dysfunction and even disability in the late stages. OA-related pain and dysfunction increase the risk of mortality [3] as well as the societal economic burden [4]. To address the health issue, most guidelines recommend the use of nonsteroidal antiinflammatory drugs (NSAIDs), duloxetine, or tramadol for nonoperative treatment of $\mathrm{OA}$ [2]. However, the use of these drugs is limited by tolerability and safety concerns [5].

Previous literature has confirmed that the proinflammatory cytokines, Interleukin- $1 \alpha$, and $1 \beta$ (IL- $1 \alpha / \beta)$ are pain 
mediators and play an important role in the pathogenesis of OA $[6,7]$. Inactive IL- $1 \alpha$ is stored in the cell or on the cell membrane. Once the cells are damaged, IL- $1 \alpha$ is activated and released, inducing the activation of IL- $1 \beta$, and finally promoting the progression of OA. $[8,9]$. IL- $1 \alpha$ and IL- $1 \beta$ both bind to the IL- 1 receptor 1 (IL-1R1), causing joint pain, inflammation, cartilage destruction, and bone resorption [10-13]. In addition, researchers have found that the concentration of IL-1 in the serum and joint fluid of patients with $\mathrm{OA}$ is elevated $[14,15]$. Subsequently, numerous IL-1R antagonists and IL-1R1 antibodies have been developed. However, clinical trials utilizing them in patients with $\mathrm{OA}$ did not report the desired results $[16,17]$. Lutikizumab is a new anti-IL- $1 \alpha / \beta$ dual variable domain immunoglobulin that simultaneously binds and inhibits IL- $1 \alpha$ and IL- $1 \beta$ without interfering with other IL-1 family members such as IL$1 \mathrm{Ra}$ [18]. Multiple animal experiments and clinical trials already have shown the potential of lutikizumab for the treatment of OA [19-21].

To comprehensively assess the clinical efficacy, including pain reduction and physical function improvement and the safety of lutikizumab for the treatment of OA, we designed and conducted a Bayesian network meta-analysis. Ten drugs widely used clinically were included in the meta-analysis. Based on these drugs' activity mechanism, we divided them into five groups: anti-Interleukin- $1 \alpha / \beta$ dual variable domain immunoglobulins (lutikizumab), selective Cox-2 inhibitors (celecoxib and etoricoxib), duloxetine, opioid (tramadol), and traditional NSAIDs (ibuprofen, naproxen, diclofenac, and paracetamol/acetaminophen).

\section{Method}

2.1. Literature Search. We conducted a systematic search of the PubMed, CNKI, EMBASE, and Web of Science databases, from January 2000 to January 2020, with the search terms consisted of (("Lutikizumab" OR "anti-Interleukin$1 \alpha / \beta$ dual variable domain immunoglobulin" OR "anti-Interleukin-1 $\alpha / \beta$ ”) OR ("selective cox-2 inhibitor" OR “cox-2 inhibitor" OR "etoricoxib" OR "celecoxib”) OR ("NSAIDs" OR "non-steroidal anti-inflammatory drugs" OR "acetaminophen" OR "diclofenac" OR "naproxen” OR "paracetamol” OR “ibuprofen”) OR (“duloxetine”) OR (“opioids” OR "Tramadol") AND ("osteoarthritis" OR "degenerative joint disease" OR “OA")).

Reference lists of relevant systematic reviews and metaanalyses were also reviewed to identify additional eligible studies. Only randomized clinical trials (RCTs) were included, but no restriction was placed on the language of publication

2.2. Eligibility Criteria. The inclusion criteria were as follows: (1) Only randomized clinical trials (RCTs) with prospective parallel-group design; (2) Studies comparing the target drugs with each other or placebo in participants with OA at any joint. The exclusion criteria were as follows: (1) Doseescalation studies of only one drug; (2) Studies on postoperative patients with OA; (3) Reviews, systematic reviews and meta-analyses, case report, conference abstractions, letters, pharmacokinetical or pharmacodynamical studies, and animal experimental studies.

2.3. Quality Assessment. Two authors conducted the methodological quality and bias assessment of included studies with the Cochrane risk of the bias assessment tool strictly. The following indexes were evaluated and ranked as low risk of bias, unclear risk of bias, or high risk of bias: sequence generation, allocation concealment, blinding, incomplete outcome data, selection outcome reporting, and other sources of bias [22]. All disputes were resolved through discussion.

2.4. Data Extraction. Author, publication year, number of patients, mean age, gender ratio (male/female), diseased joint, funded or not, intervention methods, follow-up period, and outcome data were extracted from included studies. We would give priority to select the data from the intention-totreat analysis to reduce the withdrawal bias if available. For studies involving multiple treatment groups with different doses of the same drug, we selected the most effective dose group based on the respective study's recommendations [23].

2.5. Outcome Measures. The primary efficacy endpoint was pain relief, and the secondary efficacy outcome was function improvement. Considering the differences between the baseline value of each included study, which may lower the reliability of the results and conclusions, the change-frombaseline score at the last follow-up (mean \pm SD) was used to evaluate the efficacy to minimize the biases caused by heterogeneity of baseline values. No restriction was placed on the types of questionnaire used in pain evaluation. The function subscales of Western Ontario and McMaster Universities Arthritis Index (WOMAC) were used to evaluate the function improvements preferentially. Any other functional measurement scales, such as the Lequesne index, would be used if no WOMAC function score was reported. Standardised mean difference (SMD) was used because results from different scales were included in the same network [22].

The safety outcomes included the withdrawal due to adverse effects (AEs), serious AEs, and any drug-related AEs. Serious AEs included any AEs that resulted in death, was life-threatening, needed for hospitalization, or prolonged the existing hospitalization, caused disability/incapacity, or caused anomaly/birth defect. The odds ratio (OR) with $95 \%$ confidence intervals (CI) was used to measure the safety of target drugs versus placebo or against each other.

2.6. Statistical Analysis. Conventional direct meta-analyses comparing the efficacy and safety of treatments with placebo were conducted in Stata/MP (version 14.0, Stata Corp, College Station, Texas, USA). The heterogeneity across studies was tested by the $Q$ and $I^{2}$ statistic, in which $P<0.05$ or $I^{2}$ $>50 \%$ implies significantly heterogeneity. If significant heterogeneity across studies was found, a random-effects model would be used. Otherwise, a fixed-effects model would be preferred.

The random-effects Bayesian network meta-analyses were conducted in Aggregate Data Drug Information System (ADDIS, version 1.16.8). This method can augment the number of studies within each comparison and narrow the CIs' 


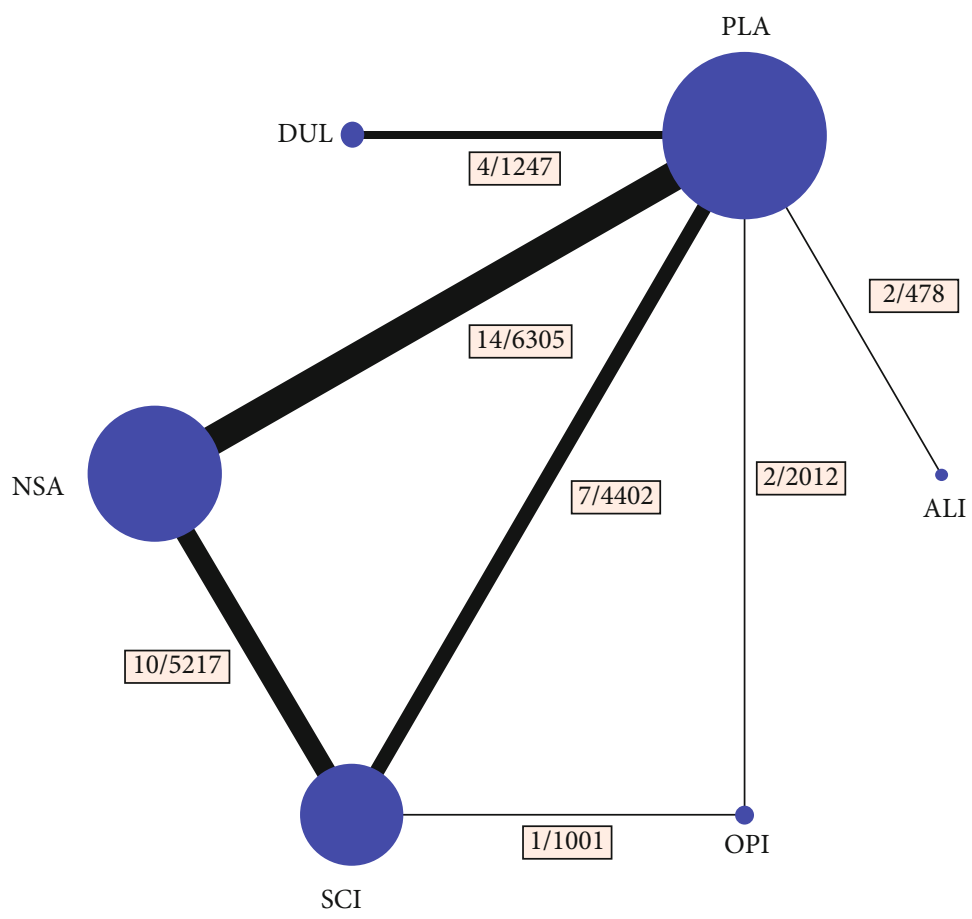

FIGURE 1: Structure of network formed by interventions. The lines between treatment nodes indicate the direct comparisons made within randomized controlled trials. Numbers $(n / n)$ near the line indicate "number of trials/number of participants" of the related comparisons.

width, and then increase the reliability of result and conclusion [24-27]. Noninformative uniform and normal prior distributions were used in this study, then four different sets of starting values were set to fit the model to yield 40000 iterations (10000 per chain) and obtain the posterior distributions of model parameters $[28,29]$. The thinning interval was set at 20 and the burn-ins at 1000 for each chain. Convergence of iterations was assessed using the Gelman-Rubin-Brooks statistic. Consistency of the network meta-analysis was reconfirmed via global inconsistency tests and node-split tests in Stata/MP (version 14.0). SMDs and ORs with 95\% CI would be generated from the posterior distribution medians. Significant differences were considered between treatments being compared when the corresponding 95\% CI did not contain 0 for the SMD or 1 for OR. Surface under the cumulative ranking (SUCRA) and the cluster-ranking plots were used to rank the efficacy and safety of different treatments. $P<$ 0.05 was considered statistically significant.

The following subgroup analyses would be performed if available: according to the drug delivery route (topical, oral, or injective) and according to the diseased joint (hip, knee, hand, or ankle).

\section{Results}

3.1. Study Selection. This network meta-analysis was conducted strictly with the Preferred Reporting Items for Systematic Reviews and Meta-Analyses (PRISMA) guidelines [30].

Twenty-four eligible studies, including 26 trials, were finally identified [31-54]. The details of the selection process are shown in Supplementary Appendix Figure 1. Six treatment arms (anti-Interleukin-1 $\alpha / \beta$ dual variable domain immunoglobulins (ALI), selective Cox-2 inhibitors (SCI), NSAIDs (NSA), duloxetine (DUL), opioids (OPI), and placebo (PLA)) were included in the network (Figure 1).

3.2. Study Characteristics. 11858 patients were assessed in this study. Most of the 26 trials included studied knee or hip OA. Only two trials with 541 patients studied hand OA.

Across the trials, the mean age of the patients was 62.35 years (range: 58.15 to 60.00 years). The proportion of male patients was $30.22 \%$ (range: $15.31 \%$ to $54.03 \%$ ), and the median follow-up was 84 days (IQR $42-89.25$ days). The number of patients enrolled for each treatment was 326 (ALI), 2518 (SCI), 3985 (NSA), 621 (DUL), 1405 (OPI), and 3033 (placebo).

The details of patient baseline characteristics are presented in Supplementary Appendix Table 1. The methodological quality and bias-risk evaluations of all included studies are presented in Supplementary Appendix Table 2. Based on these results, the main contributing factors to risks of bias were performance bias, selection bias, and attrition bias.

\subsection{Primary Efficacy Endpoint}

3.3.1. Conventional Direct Meta-Analysis. Twenty-two trials comparing five drugs with placebo were analyzed. The random-effects model was used because of the heterogeneity of the studies and interventions.

No significant differences were found in the comparison of placebo with ALI (SMD 1.118, 95\% CI [-0.374 to 2.610], $P>0.05$ ) or OPI (SMD $1.914,95 \%$ CI $[-2.833$ to 6.660 ], $P$ $>0.05)$. The other treatments all had greater efficacy than 
placebo for pain relief. DUL had the greatest efficacy for pain relief (SMD 4.764, 95\% CI [3.895 to 5.632], $P<0.05$ ). The details of the direct meta-analyses for all treatments compared with placebo are presented in Table 1.

3.3.2. Network Meta-Analysis. Twenty-six trials were analyzed in the pain-relief network. As no significant inconsistency was reported in global inconsistency tests and nodesplit tests, the consistency model was used.

DUL was the most efficacious treatment for pain relief (SMD compared with placebo $4.76,95 \%$ CI [2.35 to 7.17]), while both ADL (SMD 1.11, 95\% CI [-2.29 to 4.52]) and OPI (SMD 1.65, 95\% CI [-1.53 to 4.83]) showed no benefit compared with placebo (Figure 2 and Table 2). According to the SUCRA value, DUL had the greatest effect on pain relief (SUCRA $=88.7 \%$ ), followed by SCI (SUCRA $=88.4 \%$ ), and lastly ALI (SUCRA $=28.6 \%$ ). The detailed results of the SUCRA rank are presented in Supplementary Appendix Table 3.

\subsection{Secondary Efficacy Endpoint}

3.4.1. Conventional Direct Meta-Analysis. Except for ALI (SMD 0.99, 95\% CI [-2.27 to 2.417], $P>0.05$ ) and OPI (SMD 1.700, 95\% CI [-2.920 to 6.320], $P>0.05$ ), all other treatments were superior to placebo. SCI had the greatest efficacy for physical function improvement (SMD 4.498, 95\% CI [2.402 to 6.594], $P<0.05)$. The details of the direct metaanalyses for all treatments compared with placebo are presented in Table 1.

3.4.2. Network Meta-Analysis. A total of 26 trials were included in the functional improvement network. No significant inconsistency was found, so the consistency model was more suitable statistically than the inconsistency model.

Similar to the results of the direct meta-analysis, nonsignificant differences were found in comparison of placebo with ALI (SMD 0.992, 95\% CI [-0.433 to 4.25]) and OPI (SMD 1.12, 95\% CI [-1.92 to 4.17]), and SCI was the most efficacious treatment for physical function improvement (SMD 3.94, 95\% CI [2.48 to 5.40]) (Figure 2 and Table 2). The results of most SUCRA rankings showed that the most efficacious treatment was SCI (SUCRA $=88.4 \%$ ), and the least effective one was ALI (SUCRA = 29.6\%) (Supplementary Appendix Table 3).

\subsection{Safety Endpoint}

3.5.1. Conventional Direct Meta-Analysis. Twenty trials involving all five therapies were analyzed in the conventional direct meta-analyses. There was no significant heterogeneity reported, and a fixed-effects model was used. DUL, NSA, and OPI had greater rates for all of safety endpoints compared with placebo, while ALI and SCI did not show a significantly higher risk for any safety endpoint. The details of the pairwise meta-analysis for all drugs compared with placebo are shown in Table 1.

3.5.2. Network Meta-Analysis. Twenty-four trials involving all five treatments were analyzed in the safety network. Node-split tests and global inconsistency tests were per- formed, and no inconsistency was reported. The consistency model was preferred rather than the inconsistency model.

No treatment had more withdrawals due to adverse events (AE), nor a higher incidence of serious AEs, nor any drug-related AEs. Based on the results of the network comparisons, SCI had the lowest rate of withdrawal due to AEs (SURCA 92.6\%, OR -0.11, 95\% CI [-0.40 to 0.17]), the lowest rate of serious AEs (SURCA 80.2\%, OR -0.01, 95\% CI [-0.70 to 0.68$]$ ), and the lowest rate of drug-related AEs (SURCA $75.5 \%$, OR $0.07,95 \%$ CI [-0.10 to 0.24$]$ ). The cluster rank plots showed that SCI was the optimum treatment from the perspective of safety and efficacy (The results of clusterrank plots can be seen in Supplementary Appendix Figure 2). The relative safety between different treatments is presented in Table 3. The SURCA and relative safety compared to placebo are presented in Supplementary Appendix Table 4.

3.6. Subgroup Analysis. Two subgroup analyses were conducted.

The first subgroup analysis conducted exploited the impacts of different drug delivery routes. Three of the 26 trials used topical drug delivery methods. After excluding these studies, no substantial change was revealed. DUL had the largest efficacy for pain relief (SMD 4.76, 95\% CI [2.33 to 7.19]), and SCI for functional improvement (SMD 4.19, 95\% CI [2.70 to 5.68]). No treatment showed a higher risk of any safety endpoint (Supplementary Appendix Table 5).

In the second subgroup analysis, there were only two trials on hand joints while others all studied knee or hip joints. After excluding these studies, no substantial change was reported. Similarly, DUL had the highest effect for pain relief (SMD 4.76, 95\% CI [2.32 to 7.20]), and SCI for functional improvement (SMD 4.07, 95\% CI [2.59 to 5.55]). No treatment showed a higher risk of any safety endpoint (Supplementary Appendix Table 5).

\section{Discussion}

This is the first network meta-analysis comparing the efficacy and safety of lutikizumab, the new anti-Interleukin- $1 \alpha / \beta$ dual variable domain immunoglobulin, for treating OA with drugs recommended by guidelines [2]. We included all available evidence from randomized clinical trials (RCTs) directly or indirectly comparing lutikizumab with traditional treatments for OA and used the Bayesian method to increase the number of comparisons to enhance the power of the study. As mentioned above, considering the difference in the baseline values from different study populations and their influence on the results, we chose the change-from-baseline score as the outcome measure and only included the literature that reported the results of the change-from-baseline score. Our main findings are (1) ALI (lutikizumab) is not associated with pain relief or functional improvement of OA compared with placebo; (2) DUL, SCI, and NSA therapies all can improve every symptom of OA effectively and have a significant advantage over OPI and ALI; (3) SCI, ALI, DUL, NSA, and OPI are tolerated well for patients in long-term treatment of OA compared with placebo. These 


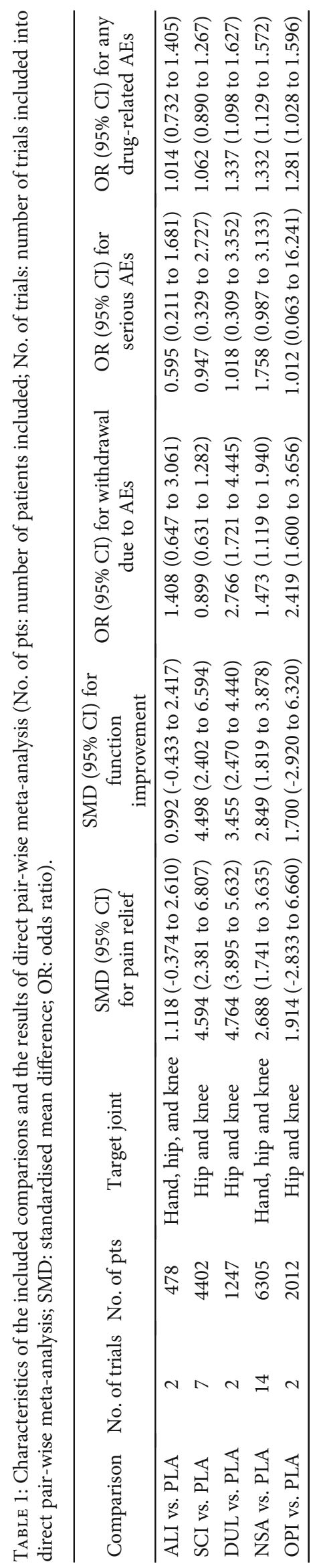




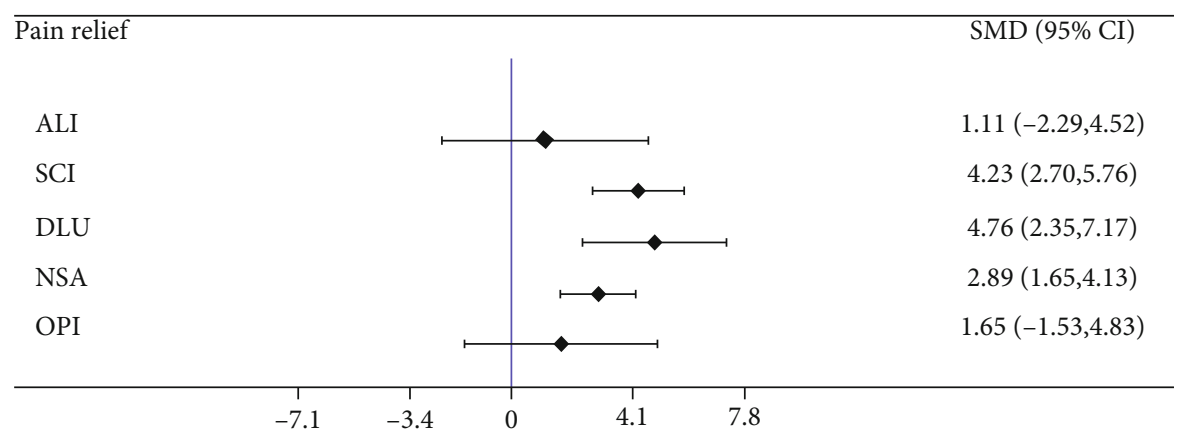

Function improvement

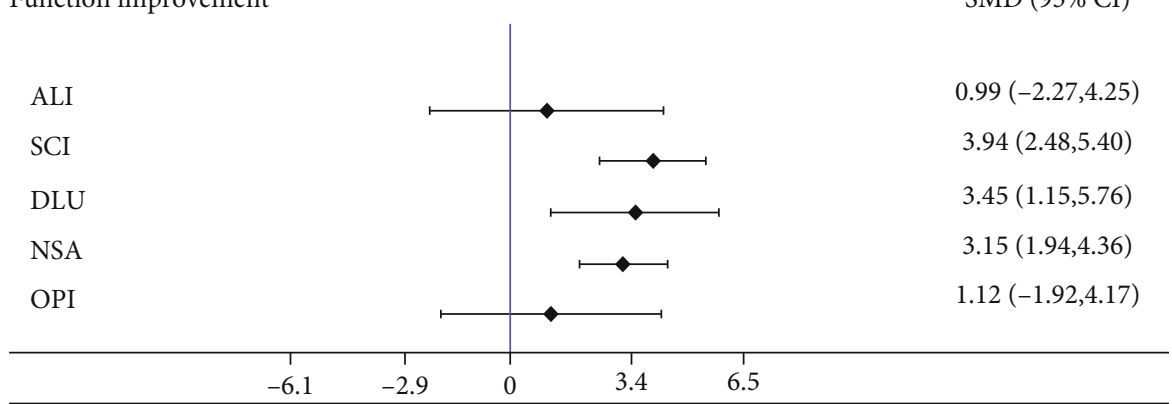

Figure 2: The forest plots. The forest plots of pain relief and function improvement for network meta-analysis. (SMD: standardised mean difference; CI: confidence interval).

TABLE 2: Detailed results of network meta-analysis for pain (Bold) and function (Italic) (Data are standardised mean difference, from the top left to the bottom right, higher comparator vs. lower comparator, and their related 95\% CI).

\begin{tabular}{|c|c|c|c|c|c|}
\hline ALI & 2.95 (-0.62 to 6.53$)$ & $2.46(-1.53$ to 6.45$)$ & $2.16(-1.31$ to 5.64$)$ & $0.14(-4.32$ to 4.60$)$ & $-0.99(-4.25$ to 2.27$)$ \\
\hline$-3.11(-6.85$ to 0.62$)$ & SCI & $-0.49(-3.22$ to 2.24$)$ & $-0.79(-2.18$ to 0.59$)$ & $-2.82(-6.01$ to 0.37$)$ & $-3.94(-5.40$ to -2.48$)$ \\
\hline$-3.65(-7.82$ to 0.53$)$ & $-0.53(-3.39$ to 2.33$)$ & DLU & $-0.30(-2.91$ to 2.30$)$ & $-2.33(-6.14$ to 1.49$)$ & $-3.45(-5.76$ to -1.15$)$ \\
\hline$-1.77(-5.40$ to 1.85$)$ & $1.34(-0.07$ to 2.76$)$ & $1.87(-0.84$ to 4.59$)$ & NSA & $-2.02(-5.23$ to 1.18$)$ & $-3.15(-4.36$ to -1.94$)$ \\
\hline$-0.54(-5.20$ to 4.13$)$ & $2.58(-0.76$ to 5.91$)$ & $3.11(-0.89$ to 7.10$)$ & $1.23(-2.10$ to 4.57$)$ & OPI & $-1.12(-4.17$ to 1.92$)$ \\
\hline $1.11(-2.29$ to 4.52$)$ & $4.23(2.70$ to 5.76$)$ & $4.76(2.35$ to 7.17$)$ & $2.89(1.65$ to 4.13$)$ & $1.65(-1.53$ to 4.83$)$ & PLA \\
\hline
\end{tabular}

results indicate that lutikizumab is not suitable for the treatment of OA because it provides no improvement in joint pain and dysfunction, while selective Cox-2 inhibitors (such as celecoxib and etoricoxib) are the ideal choice for the treatment of OA from the perspective of safety and efficacy. Combined with the results from other clinical trials $[16,17]$, the inhibition of IL- $\alpha / \beta$ does not seem to be a new way to treat $\mathrm{OA}$ in the future.

There are several limitations in this study. Considering the unmanageable confounding factors in non-RCTs and their unpredictable influences on the results of network meta-analysis, only RCTs were included. Nevertheless, nonRCTs, especially observational studies, can provide valuable insight into the long-term effectiveness and safety of treatment for OA. To enhance the credibility of this meta-analysis, only high-quality studies were included. This may have contributed to the small number of studies included. Publication bias could be a significant problem for this study, especially the funnel plots that showed a dubious asymmetry. We tried to adjust the publication bias using the trimming and filling method. However, a previous study suggested that the results of the trimming and filling method should be interpreted as a sensitivity analysis rather than a corrected estimate of publication bias [55]. So, the results of this study should be interpreted cautiously, particularly for ALI in which the number of included studies is smaller compared with other treatments. Although we have conducted two subgroup analyses to reduce the impact of potential confounding factors, there are still many other factors that could affect the reliability of the results, such as the differences in comorbidities, duration of $\mathrm{OA}$, and grade of $\mathrm{OA}$ in the study populations. For instance, comorbidities usually cause worse symptom management and consequentially affect the results of analgesic effectiveness assessment. Paradoxically, research on analgesics often excludes people with clinically significant comorbidities and does not systematically describe the distribution of comorbidities in the study population. Most of the included studies, coincidentally, failed to report an accurate grade or duration of OA. We were unable to adjust for these factors because of the insufficiency of the related data, and thus those results should be interpreted with caution. More high-quality trials are needed. 


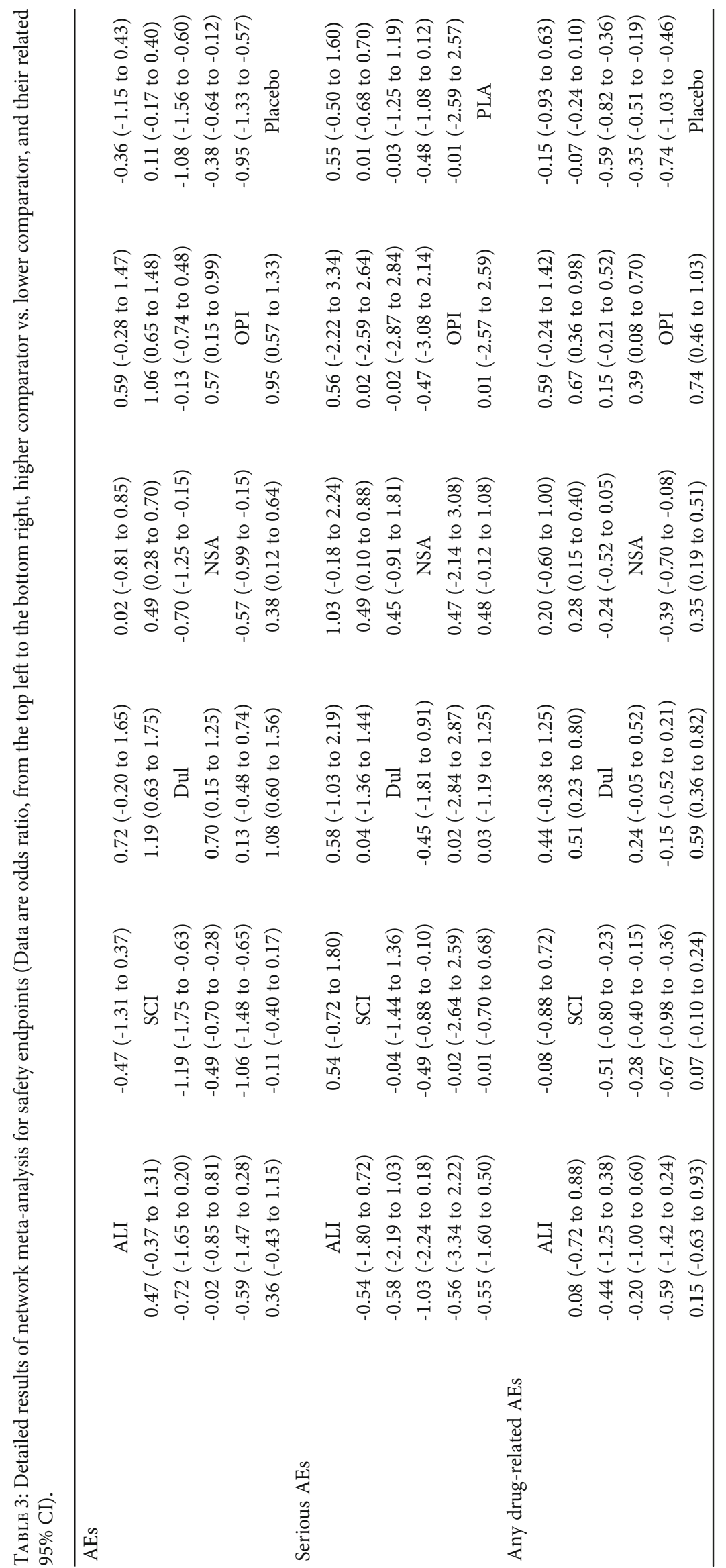




\section{Conclusion}

24 studies, involving 26 trials assessing 11858 patients, were included in this network meta-analysis. The results show that lutikizumab, the new anti-Interleukin- $1 \alpha / \beta$ dual variable domain immunoglobulin, did not improve pain or function in the comparison with placebo. Selective cox- 2 inhibitors and duloxetine remain the most effective and safest treatment for OA. More high-quality trials are needed to reconfirm the findings of this study.

\section{Data Availability}

Data generated or analyzed during this study are included in this published article. The datasets generated during and/or analyzed during the current study are available from the corresponding author on reasonable request.

\section{Ethical Approval}

This article does not contain any studies with human participants or animals performed by any of the authors.

\section{Disclosure}

The study funders/sponsors had no role in the design and conduct of the study; collection, management, analysis, and interpretation of the data; preparation, review, or approval of the manuscript; and decision to submit the manuscript for publication.

\section{Conflicts of Interest}

The authors declare that they do not have any competing interests.

\section{Authors' Contributions}

Pengcheng Dou and Ren Wu conceived the study, participated in its design and coordination, and critically revised the manuscript. Ziqin Cao, Wanchun Wang, and Shuo Jie had full access to all of the data collection, analysis, and interpretation. Ziqin Cao and Yajia Li drafted the manuscript. Jian Zhou and Xuantao $\mathrm{Hu}$ were study investigators and contributed to the process of data collection. Yihan Li and Zeling Long contributed to the process of study research and selection. Tong $\mathrm{Wu}$ and Dilihumaer Aili contributed to the risk of bias assessment. All authors read and approved the final manuscript.

\section{Acknowledgments}

The authors would like to express their gratitude to EditSprings (https://www.editsprings.com/) for the expert linguistic services provided. This work was supported by the Mittal Innovation Project of Central South University (Grant No. GCX20190879Y) and the Fundamental Research Funds for the Central Universities of Central South University (Grant No. 2018zzts930).

\section{Supplementary Materials}

The Supplementary Material includes 5 supplementary appendix tables and 2 supplementary appendix figures which support our findings. (Supplementary Materials)

\section{References}

[1] C. B. Johannes, T. K. Le, X. Zhou, J. A. Johnston, and R. H. Dworkin, "The prevalence of chronic pain in United States adults: results of an Internet-based survey," The Journal of Pain, vol. 11, no. 11, pp. 1230-1239, 2010.

[2] S. L. Kolasinski, T. Neogi, M. C. Hochberg et al., "2019 American College of Rheumatology/Arthritis Foundation Guideline for the management of osteoarthritis of the hand, hip, and knee," Arthritis \& Rhematology, vol. 72, no. 2, pp. 220-233, 2020.

[3] E. Nuesch, P. Dieppe, S. Reichenbach, S. Williams, S. Iff, and P. Juni, "All cause and disease specific mortality in patients with knee or hip osteoarthritis: population based cohort study," BMJ, vol. 342, no. mar08 2, article d1165, 2011.

[4] Institute of Medicine US Committee on Advancing Pain Research CA, Education, Relieving pain in America: a blueprint for transforming prevention, care, education, and research, National Academies Press (US), Washington (DC), 2011.

[5] S. Glyn-Jones, A. J. R. Palmer, R. Agricola et al., "Osteoarthritis,” LANCET, vol. 386, no. 9991, pp. 376-387, 2015.

[6] C. M. de Oliveira, R. K. Sakata, A. M. Issy, L. R. Gerola, and R. Salomão, "Cytokines and pain," Revista Brasileira de Anestesiologia, vol. 61, no. 2, pp. 255-265, 2011.

[7] M. Kapoor, J. Martel-Pelletier, D. Lajeunesse, J. P. Pelletier, and H. Fahmi, "Role of proinflammatory cytokines in the pathophysiology of osteoarthritis," Nature Reviews Rheumatology, vol. 7, no. 1, pp. 33-42, 2011.

[8] C. Garlanda, C. A. Dinarello, and A. Mantovani, "The interleukin-1 family: back to the future," Immunity, vol. 39, no. 6, pp. 1003-1018, 2013.

[9] G. Schett, J. M. Dayer, and B. Manger, "Interleukin-1 function and role in rheumatic disease," Nature Reviews Rheumatology, vol. 12, no. 1, pp. 14-24, 2016.

[10] R. E. Miller, R. J. Miller, and A. M. Malfait, "Osteoarthritis joint pain: the cytokine connection," Cytokine, vol. 70, no. 2, pp. 185-193, 2014.

[11] J. Lorenzo, M. Horowitz, and Y. Choi, “Osteoimmunology: interactions of the bone and immune system," Endocrine Reviews, vol. 29, no. 4, pp. 403-440, 2008.

[12] F. M. Brennan and I. B. McInnes, "Evidence that cytokines play a role in rheumatoid arthritis," The Journal of Clinical Investigation, vol. 118, no. 11, pp. 3537-3545, 2008.

[13] M. B. Goldring, J. Birkhead, L. J. Sandell, T. Kimura, and S. M. Krane, "Interleukin 1 suppresses expression of cartilage-specific types II and IX collagens and increases types I and III collagens in human chondrocytes," The Journal of Clinical Investigation, vol. 82, no. 6, pp. 2026-2037, 1988.

[14] C. A. Towle, H. H. Hung, L. J. Bonassar, B. V. Treadwell, and D. C. Mangham, "Detection of interleukin-1 in the cartilage of patients with osteoarthritis: a possible autocrine/paracrine role in pathogenesis," Osteoarthritis and Cartilage, vol. 5, no. 5, pp. 293-300, 1997. 
[15] M. N. Farahat, G. Yanni, R. Poston, and G. S. Panayi, "Cytokine expression in synovial membranes of patients with rheumatoid arthritis and osteoarthritis," Annals of the Rheumatic Diseases, vol. 52, no. 12, pp. 870-875, 1993.

[16] X. Chevalier, P. Goupille, A. D. Beaulieu et al., "Intraarticular injection of anakinra in osteoarthritis of the knee: a multicenter, randomized, double-blind, placebo-controlled study," Arthritis and Rheumatism, vol. 61, no. 3, pp. 344-352, 2009.

[17] S. B. Cohen, S. Proudman, A. J. Kivitz et al., "A randomized, double-blind study of AMG 108 (a fully human monoclonal antibody to IL-1R1) in patients with osteoarthritis of the knee," Arthritis Research \& Therapy, vol. 13, no. 4, 2011.

[18] S. E. Lacy, C. Wu, D. J. Ambrosi et al., "Generation and characterization of ABT-981, a dual variable domain immunoglobulin (DVD- $\operatorname{Ig}^{\mathrm{TM}}$ ) molecule that specifically and potently neutralizes both IL- $1 \alpha$ and IL-1 $\beta$," $m A b s$, vol. 7, no. 3, pp. 605-619, 2015.

[19] R. V. H. M. C. D. Kamath, M. Hart, D. Conlon, and T. Ghayur, "126 Simultaneous targeting of IL-1A and IL-1B by a dualvariable-domain immunoglobulin (DVD-IG(tm)) prevents cartilage degradation in preclinical models of osteoarthritis," Osteoarthritis and Cartilage, vol. 19, 2011.

[20] R. V. S. G. Z. C. Kamath, G. Simler, C. Zhou et al., "Blockade of both IL-1A and IL-1B by a combination of monoclonal antibodies prevents the development and reverses established pain in a preclinical model of osteoarthritis," Osteoarthritis and Cartilage, vol. 20, SUPPLEMENT 1, 2012.

[21] S. X. Wang, S. B. Abramson, M. Attur et al., "Safety, tolerability, and pharmacodynamics of an anti-interleukin- $1 \alpha / \beta$ dual variable domain immunoglobulin in patients with osteoarthritis of the knee: a randomized phase 1 study," Osteoarthritis and Cartilage, vol. 25, no. 12, pp. 1952-1961, 2017.

[22] J. P. Higgins, D. G. Altman, P. C. Gotzsche et al., "The Cochrane Collaboration's tool for assessing risk of bias in randomised trials," BMJ, vol. 343, no. oct18 2, article d5928, 2011.

[23] DJAD and HJ, "Chapter 16: Special topics in statistics," in Cochrane Handbook for Systematic Reviews of Interventions, H. JPT and S. Green, Eds., The Cochrane Collaboration, 2011, Version 5.1.0 [updated March 2011].

[24] H. C. Bucher, G. H. Guyatt, L. E. Griffith, and S. D. Walter, "The results of direct and indirect treatment comparisons in meta-analysis of randomized controlled trials," Journal of Clinical Epidemiology, vol. 50, no. 6, pp. 683-691, 1997.

[25] D. M. Caldwell, A. E. Ades, and J. P. Higgins, "Simultaneous comparison of multiple treatments: combining direct and indirect evidence," BMJ, vol. 331, no. 7521, pp. 897-900, 2005.

[26] D. M. Caldwell, N. J. Welton, and A. E. Ades, "Mixed treatment comparison analysis provides internally coherent treatment effect estimates based on overviews of reviews and can reveal inconsistency," Journal of Clinical Epidemiology, vol. 63, no. 8, pp. 875-882, 2010.

[27] G. Lu and A. E. Ades, "Combination of direct and indirect evidence in mixed treatment comparisons," Statistics in Medicine, vol. 23, no. 20, pp. 3105-3124, 2004.

[28] A. E. Ades, M. Sculpher, A. Sutton et al., "Bayesian methods for evidence synthesis in cost-effectiveness analysis," PharmacoEconomics, vol. 24, no. 1, pp. 1-19, 2006.

[29] A. Sutton, A. E. Ades, N. Cooper, and K. Abrams, "Use of indirect and mixed treatment comparisons for technology assessment," PharmacoEconomics, vol. 26, no. 9, pp. 753767, 2008.
[30] PRISMA-P Group, D. Moher, L. Shamseer et al., "Preferred reporting items for systematic review and meta-analysis protocols (PRISMA-P) 2015 statement," Systematic Reviews, vol. 4, no. 1,2015

[31] J. Zacher, D. Feldman, R. Gerli et al., "A comparison of the therapeutic efficacy and tolerability of etoricoxib and diclofenac in patients with osteoarthritis," Current Medical Research and Opinion, vol. 19, no. 8, pp. 725-736, 2008.

[32] A. Puopolo, J. A. Boice, J. L. Fidelholtz et al., "A randomized placebo-controlled trial comparing the efficacy of etoricoxib $30 \mathrm{mg}$ and ibuprofen $2400 \mathrm{mg}$ for the treatment of patients with osteoarthritis," Osteoarthritis Cartilage, vol. 15, no. 12, pp. 1348-1356, 2007.

[33] M. J. Prior, D. D. Harrison, and M. E. Frustaci, “A randomized, double-blind, placebo-controlled 12 week trial of acetaminophen extended release for the treatment of signs and symptoms of osteoarthritis," Current Medical Research and Opinion, vol. 30, no. 11, pp. 2377-2387, 2014.

[34] P. Emery, T. Koncz, S. Pan, and S. Lowry, "Analgesic effectiveness of celecoxib and diclofenac in patients with osteoarthritis of the hip requiring joint replacement surgery: a 12-week, multicenter, randomized, double-blind, parallel-group, doubledummy, noninferiority study," Clinical Therapeutics, vol. 30, no. 1, pp. 70-83, 2008.

[35] D. B. McKenna and H. Wendt, "Celecoxib versus diclofenac in the management of osteoarthritis of the knee: a placebo-controlled, randomised, double-blind comparison," Scandinavian Journal of Rheumatology, vol. 30, no. 1, pp. 11-18, 2001.

[36] R. D. Altman, R. L. Dreiser, C. L. Fisher, W. F. Chase, D. S. Dreher, and J. Zacher, "Diclofenac sodium gel in patients with primary hand osteoarthritis: a randomized, double-blind, placebo-controlled trial," The Journal of Rheumatology, vol. 36, no. 9, pp. 1991-1999, 2009.

[37] A. A. M. Bookman, "Effect of a topical diclofenac solution for relieving symptoms of primary osteoarthritis of the knee: a randomized controlled trial," Canadian Medical Association Journal, vol. 171, no. 4, pp. 333-338, 2004.

[38] A. Gibofsky, M. C. Hochberg, M. J. Jaros, and C. L. Young, "Efficacy and safety of low-dose submicron diclofenac for the treatment of osteoarthritis pain: a 12 week, phase 3 study," Current Medical Research and Opinion, vol. 30, no. 9, pp. 1883-1893, 2014.

[39] M. N. Essex, M. A. O'Connell, R. Behar, and W. Bao, "Efficacy and safety of nonsteroidal anti-inflammatory drugs in Asian patients with knee osteoarthritis: summary of a randomized, placebo-controlled study," International Journal of Rheumatic Diseases, vol. 19, no. 3, pp. 262-270, 2016.

[40] K. Reed, A. Collaku, and S. Moreira, "Efficacy and safety of twice daily sustained-release paracetamol formulation for osteoarthritis pain of the knee or hip: a randomized, doubleblind, placebo-controlled, twelve-week study," Current Medical Research and Opinion, vol. 34, no. 4, pp. 689-699, 2018.

[41] M. N. Essex, P. Bhadra, and G. H. Sands, "Efficacy and tolerability of celecoxib versus naproxen in patients with osteoarthritis of the knee: a randomized, double-blind, doubledummy trial," Journal of International Medical Research, vol. 40, no. 4, pp. 1357-1370, 2012.

[42] A. T. Leung, K. Malmstrom, A. E. Gallacher et al., "Efficacy and tolerability profile of etoricoxib in patients with osteoarthritis: a randomized, double-blind, placebo and activecomparator controlled 12-week efficacy trial," Current Medical Research and Opinion, vol. 18, no. 2, pp. 49-58, 2002. 
[43] A. C. Gordo, C. Walker, B. Armada, and D. Zhou, "Efficacy of celecoxib versus ibuprofen for the treatment of patients with osteoarthritis of the knee: a randomized double-blind, noninferiority trial," Journal of International Medical Research, vol. 45, no. 1, pp. 59-74, 2017.

[44] F. U. Niethard, M. S. Gold, G. S. Solomon et al., "Efficacy of topical diclofenac diethylamine gel in osteoarthritis of the knee," The Journal of Rheumatology, vol. 32, no. 12, pp. 2384-2392, 2005.

[45] B. P. DeLemos, J. Xiang, C. Benson et al., "Tramadol hydrochloride extended-release once-daily in the treatment of osteoarthritis of the knee and/or hip: a double-blind, randomized, dose-ranging trial," American Journal of Therapeutics, vol. 18, no. 3, pp. 216-226, 2011.

[46] J. Y. Reginster, K. Malmstrom, A. Mehta et al., "Evaluation of the efficacy and safety of etoricoxib compared with naproxen in two, 138-week randomised studies of patients with osteoarthritis," Annals of the Rheumatic Diseases, vol. 66, no. 7, pp. 945-951, 2007.

[47] T. J. Gana, M. L. Pascual, R. R. Fleming et al., "Extendedrelease tramadol in the treatment of osteoarthritis: a multicenter, randomized, double-blind, placebo-controlled clinical trial," Current Medical Research and Opinion, vol. 22, no. 7, pp. 1391-1401, 2006.

[48] O. Svensson, M. Malmenäs, L. Fajutrao, E. M. Roos, and L. S. Lohmander, "Greater reduction of knee than hip pain in osteoarthritis treated with naproxen, as evaluated by WOMAC and SF-36," Annals of the Rheumatic Diseases, vol. 65, no. 6, pp. 781-784, 2006

[49] R. M. Fleischmann, H. Bliddal, F. J. Blanco et al., "A PhaseIITrial of lutikizumab, an anti-interleukin- $1 \alpha / \beta$ dual variable domain immunoglobulin, in knee osteoarthritis patients with synovitis," Arthritis \& Rheumatology, vol. 71, no. 7, pp. 1056-1069, 2019.

[50] M. Kloppenburg, C. Peterfy, I. K. Haugen et al., "Phase IIa, placebo-controlled, randomised study of lutikizumab, an antiinterleukin- $1 \alpha$ and anti-interleukin- $1 \beta$ dual variable domain immunoglobulin, in patients with erosive hand osteoarthritis," Annals of the Rheumatic Diseases, vol. 78, no. 3, pp. 413-420, 2019.

[51] A. S. Chappell, D. Desaiah, H. Liu-Seifert et al., "A doubleblind, randomized, placebo-controlled study of the efficacy and safety of duloxetine for the treatment of chronic pain due to osteoarthritis of the knee," Pain Practice, vol. 11, no. 1, pp. 33-41, 2011.

[52] Y. Uchio, H. Enomoto, L. Alev et al., “A randomized, doubleblind, placebo-controlled Phase III trial of duloxetine in Japanese patients with knee pain due to osteoarthritis," Journal of Pain Research, vol. 11, pp. 809-821, 2018.

[53] A. S. Chappell, M. J. Ossanna, H. Liu-Seifert et al., "Duloxetine, a centrally acting analgesic, in the treatment of patients with osteoarthritis knee pain: a 13-week, randomized, placebocontrolled trial," PAIN, vol. 146, no. 3, pp. 253-260, 2009.

[54] G. Wang, L. Bi, X. Li et al., "Efficacy and safety of duloxetine in Chinese patients with chronic pain due to osteoarthritis: a randomized, double-blind, placebo-controlled study," Osteoarthritis Cartilage, vol. 25, no. 6, pp. 832-838, 2017.

[55] S. Duval and R. Tweedie, "Trim and fill: a simple funnel-plotbased method of testing and adjusting for publication bias in meta-analysis," Biometrics, vol. 56, no. 2, pp. 455-463, 2000. 